\title{
Automatic entry point planning for robotic post-mortem CT- based needle placement
}

\author{
Lars C. Ebert ${ }^{1}$ - Martin Fürst ${ }^{2}$ - Wolfgang Ptacek ${ }^{2}$ Thomas D. Ruder ${ }^{1,3}$. \\ Dominic Gascho $^{1}$ - Wolf Schweitzer ${ }^{1} \cdot$ Michael J. Thali ${ }^{1} \cdot$ Patricia M. Flach $^{1,4}$
}

Accepted: 2 July 2016/Published online: 15 July 2016

(C) Springer Science+Business Media New York 2016

\begin{abstract}
Introduction Post-mortem computed tomography guided placement of co-axial introducer needles allows for the extraction of tissue and liquid samples for histological and toxicological analyses. Automation of this process can increase the accuracy and speed of the needle placement, thereby making it more feasible for routine examinations. To speed up the planning process and increase safety, we developed an algorithm that calculates an optimal entry point and end-effector orientation for a given target point, while taking constraints such as accessibility or bone collisions into account.

Technique The algorithm identifies the best entry point for needle trajectories in three steps. First, the source CT data is prepared and bone as well as surface data are extracted and optimized. All vertices of the generated surface polygon are considered to be potential entry points. Second, all surface points are tested for validity within the defined hard constraints (reachability, bone collision as well as collision with other needles) and removed if invalid. All remaining
\end{abstract}

Lars C. Ebert

lars.ebert@virtopsy.com

1 Department of Forensic Medicine and Imaging, Zurich Institute of Forensic Medicine, University of Zurich, Winterthurerstrasse 190/52, 8057 Zurich, Switzerland

2 Austrian Center for Medical Innovation and Technology (ACMIT), ACMIT GmbH, Viktor Kaplan Straße 2, 2700 Wiener Neustadt, Austria

3 Institute of Diagnostic, Interventional and Pediatric Radiology, University Hospital Bern, Freiburgstrasse, 3010 Bern, Switzerland

4 Institute of Diagnostic and Interventional Radiology, University Hospital Zurich, Raemistrasse 100, 8091 Zurich, Switzerland vertices are reachable entry points and are rated with respect to needle insertion angle. Third, the vertex with the highest rating is selected as the final entry point, and the best end-effector rotation is calculated to avoid collisions with the body and already set needles.

Discussion In most cases, the algorithm is sufficiently fast with approximately 5-6 s per entry point. This is the case if there is no collision between the end-effector and the body. If the end-effector has to be rotated to avoid collision, calculation times can increase up to $24 \mathrm{~s}$ due to the inefficient collision detection used here. In conclusion, the algorithm allows for fast and facilitated trajectory planning in forensic imaging.

Keywords Virtopsy · Forensic imaging · Post-mortem needle placement $\cdot$ Biopsy $\cdot$ Trajectory planning

\section{Introduction}

Post-mortem computed tomography (PMCT) guided placement of co-axial introducer needles allows for the extraction of tissue and liquid samples for histological and toxicological analyses [1,2].

Automation of this process can increase the accuracy and speed of the needle placement, thereby making it more feasible for routine examinations [3]. A system that is able to perform automated CT-based post-mortem needle placement was presented in 2009 [4], with an updated version described in 2012, which added an automatic tool change mechanism and new trajectory planning software [5].

A linear trajectory for robotic needle placement using one of these systems requires three input parameters. First, the target needs to be defined. Depending on the required 
task (guide wire placement, liquid or tissue extraction), the needle tip will be placed in, or slightly above, the planned target point. Second, the entry point on the skin has to be defined. A number of constraints have to be considered, as insertion angle, insertion depth, bone collisions and collisions with already placed needles must be limited. Third, the final orientation (rotation around the needle axis) of the biopsy module mounted to the robot (the so called endeffector) has to be defined.

In neurosurgery and abdominal interventions, different approaches for automatic trajectory planning have been proposed [6, 7]. However, post-mortem needle placement differs from needle placement in living patients. For the safety of living patients, the actual path of the needle trajectory is essential. Poor planning or misplacement can lead to potentially life threatening complications such as hemorrhage, nerve damage, or bowl perforation. This means that, depending on the intended intervention, organs, bones, vessels, and other structures have to be segmented prior to the planning [8]. Semi-automatic or manual segmentation methods are the most flexible; however, the process of manual or semi-automatic segmentation can be time consuming [9]. While needle drivers for automatic needle placement in a clinical setting exist, to the best of our knowledge, no commercial clinical system offers automatic needle placement $[10,11]$. Instead, the task of finally placing the needle has to be achieved manually. For this purpose, robotic systems only offer a needle guide to assist with final manual placement.

In post-mortem needle placement, on the other hand, the focus lies on placement speed rather than accuracy because often needles have to be placed in multiple organs for one case (Fig. 1). Especially for institutes with high case loads, the time required for planning and executing the tissue sampling can be problematic. In addition to mechanical constraints of the robot used, the avoidance of bony structures is essential because a collision would lead to needle bending and misplacement.

According to our experience, the planning of entry points takes the most time because a position has to be found that is within reach of the robot and does not hit bones or other insurmountable anatomical structures. Especially for inexperienced users, this part tends to be challenging and time consuming. In addition, the planning of multiple needle trajectories can create complex situations that can lead to collisions between the robot endeffector and already placed needles.

To speed up the planning process and increase safety, we developed an algorithm that calculates an optimal entry point and end-effector orientation for a given target point including the avoidance of osseous obstacles and already placed needles.

\section{Technique}

\section{Imaging parameters}

For imaging, a Siemens Somatom Definition Flash (Siemens Healthcare, Erlangen, Germany) Dual Source CT was used. The standard scan performed for a post-mortem needle placement covers the thorax and abdomen and is scanned at $120 \mathrm{kVp}$ using dose modulation (reference: 400 mAs) (CAREDose 4d, Siemens Healthcare, Erlangen, Germany). It has a slice thickness of $1.5 \mathrm{~mm}$ and an increment of $1.2 \mathrm{~mm}$. Images are reconstructed using a soft kernel generating a total of 751 images.
Fig. 1 Volume reconstruction of a PMCT scan after introducer needles for tissue and liquid sampling have been placed (counter clockwise) in the right atrium of the heart, the right lung, the liver, and the right kidney as well as fat tissue

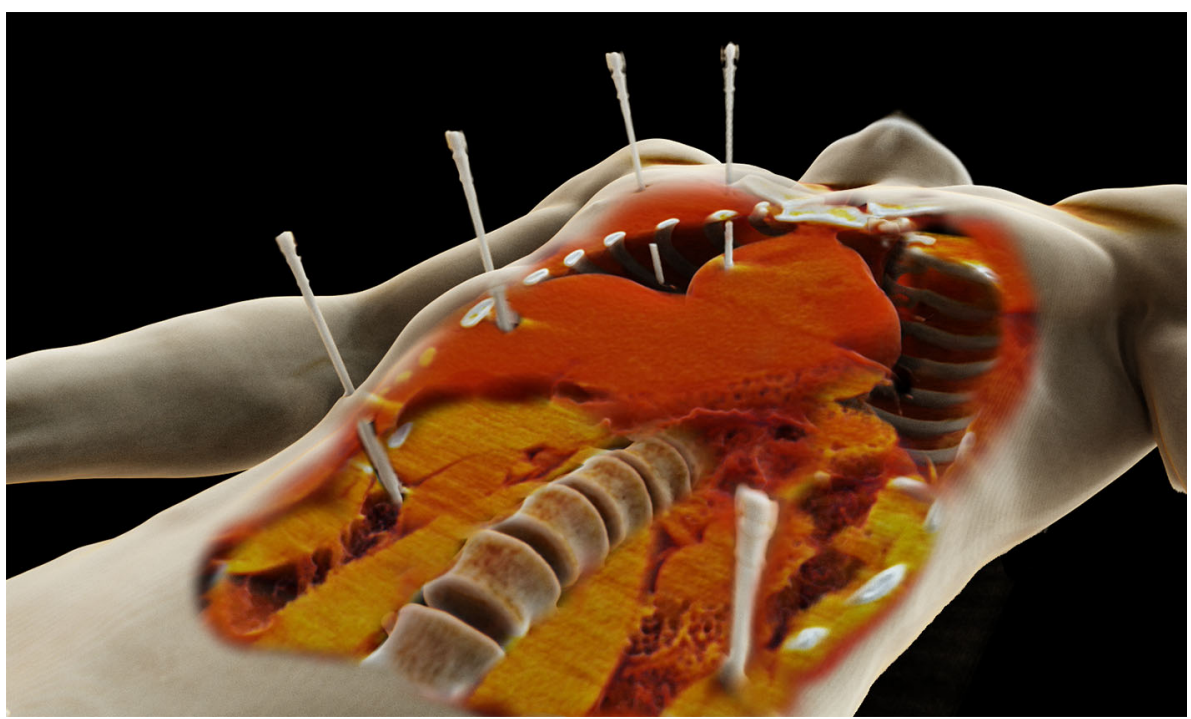




\section{Robotic arm}

A robotic arm that was mounted on a ceiling rail above the CT table and aligned in parallel was used. The system is able to automatically mount a variety of tools, one of which is a biopsy module. After mounting the biopsy tool, the robot is able to pick up coaxial introducer needles from a needle magazine and place them according to the planned trajectory. A detailed description of the system can be found in Ebert et al. [5]. An overview of the system is depicted in Fig. 2.

\section{Entry point calculation}

The presented method calculates the best valid entry point on the skin surface as well as the best rotation of the robot end-effector for a given target point inside the body for post-mortem needle placement. It is integrated into the trajectory planning software, which is based on QT 5.4 and VTK 6.3 and written in C++ using Visual Studio 2013.

The algorithm identifies the best entry point for needle trajectories in three steps (Fig. 3).

1. Data preparation: The source PMCT data is prepared, and bone as well as surface data is extracted and optimized.

2. Removal of invalid entry points: All vertices of the generated surface polygon are considered to be potential entry points and are tested for validity within the defined hard constraints (reachability, bone collision, and collision with other needles).
3. Selection of best entry point and calculation of endeffector rotation: All remaining vertices are reachable entry points and are rated with respect to needle insertion angle. The vertex with the highest rating is selected as the final entry point, and the best endeffector rotation is calculated.

\section{Data preparation}

After loading the PMCT Digital Imaging and Communication in Medicine (DICOM) dataset, it is resampled to a quarter of its original dimension. Using the resampled data, body surface and bone structures are segmented automatically. A simple thresholding filter is applied to perform the surface extraction [9]. For bone extraction, a threshold is not sufficient because either a lot of image noise is segmented as well (for lower thresholds) or the costal cartilage is left out (for higher thresholds). To overcome this issue, we used a seeded region growing filter for segmentation [12]. For automatic seed point detection, the algorithm selects all voxels with an HU above 170 on a predefined axial slice as seed points. The actual region growing filter is then carried out with a lower threshold of 95 to allow for the segmentation of costal cartilage. Based on the segmented surface and bone structures, polygon meshes are created using the marching cubes algorithm [13]. Finally, the polygon meshes are decimated to reduce the number of vertices from approximately 90,000 to 20,000. For both models, oriented bounding box (OBB) trees are generated for future collision detection [14].
Fig. 2 Robotic arm with mounted biopsy end-effector for CT-guided robotic tissue sampling. The robot is mounted onto an external axis that is parallel to the CT table to allow full access to the scanning volume of the robot

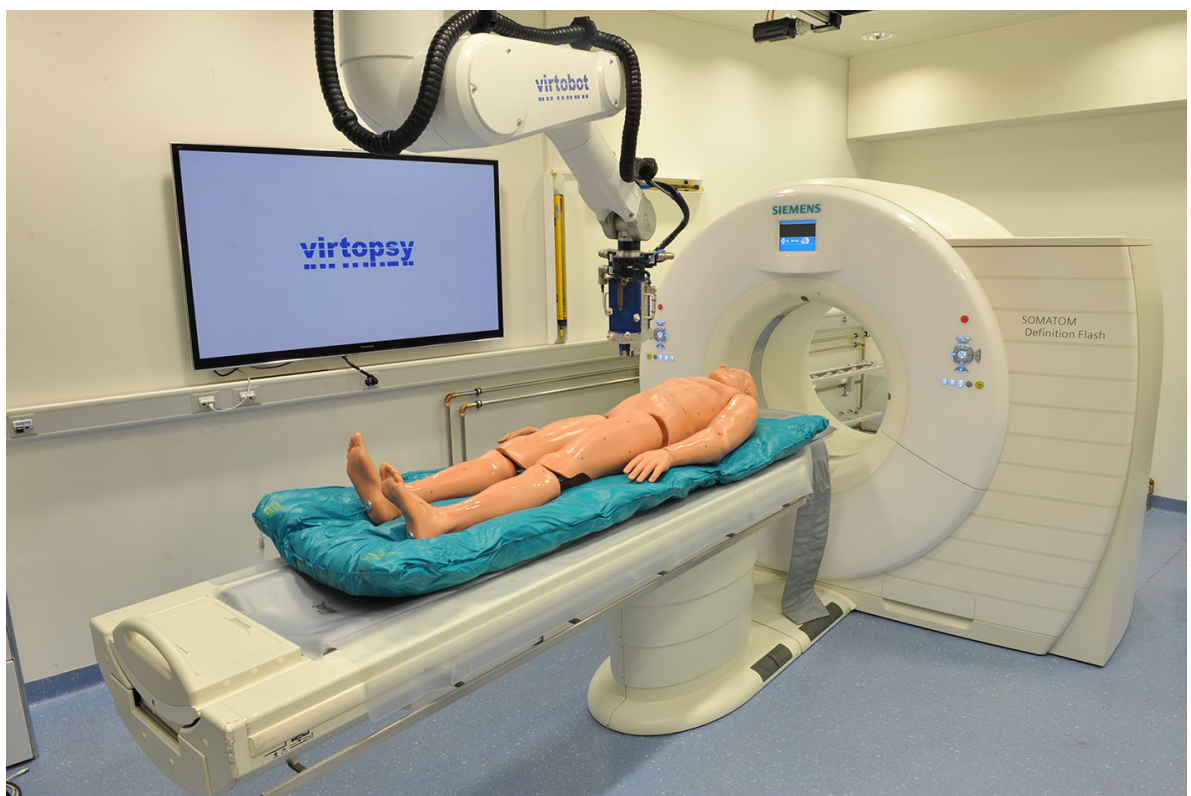


Fig. 3 Entry point planner algorithm. a Source dataset. b Extracted bone (left) and surface polygon mesh OBB trees are generated for each mesh. c Vertices that are within a needle length of the target point are marked in green. d, e Internal entry points removed. f Vertices that are not reachable by the robot due to an insertion angle larger than $40^{\circ}$ are removed. $g$ Vertices that produce trajectories with bone collisions or are closer than 3 $\mathrm{mm}$ to bones are removed.

h Final trajectory after calculating the best rotation of the end-effector
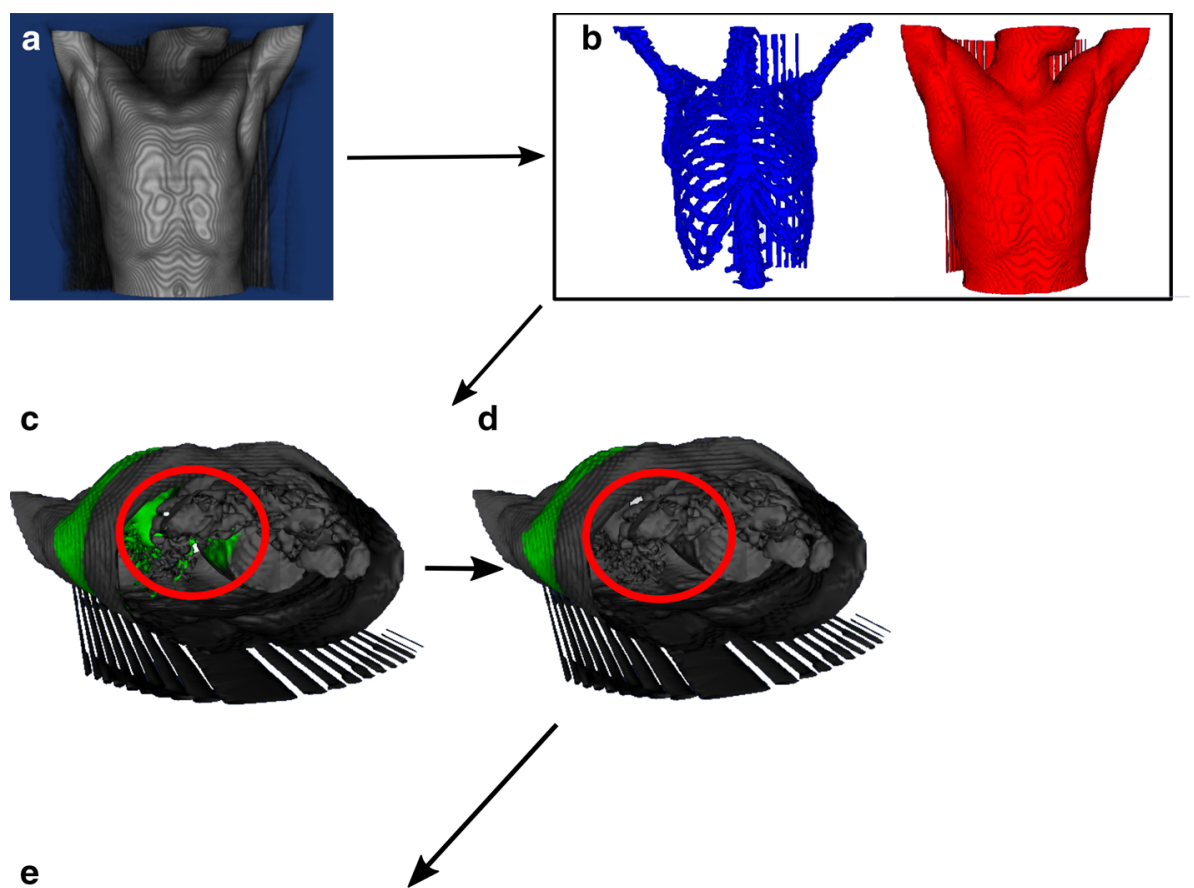

e

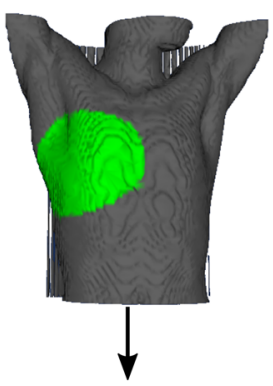

$\mathbf{f}$

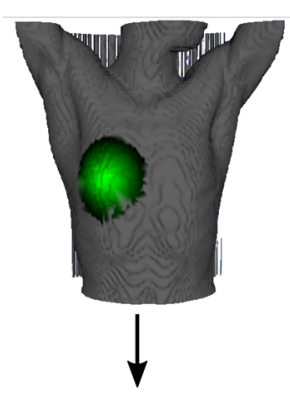

g

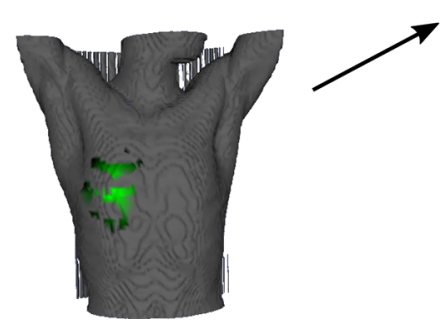

\section{Removal of invalid entry points}

All vertices of the generated surface model are considered to be potential entry points for the selected target point, but only a fraction are actually reachable. The algorithm excludes entry points that do not match the hard constraints and picks the vertex that is most perpendicular relative to the CT table as the chosen entry point. Finally, the robot end-effector rotation is calculated to avoid collisions during release motion. First, if the distance between a vertex 
and the target point is larger than the effectively usable needle length for the biopsy, the vertex is not reachable and is therefore not considered. If the distance is too small, the introducer needle would fall off after placement due to its own weight. Therefore, entry points with less than $1 / 3$ rd of the needle length inside the body are also considered to be invalid.

Surface model generation with simple thresholding means that the internal structures are also partially modeled. Because vertices that lie internally should be excluded as valid entry points, the vector between the target point and the vertex is extended and checked for collisions with the surface model. If more than one collision is present, the vertex lies within the model and is excluded.

The robotic arm has a predefined angular limit for needle insertion with respect to the axial and sagittal planes. This limitation of insertion angle was defined to minimize the chance of collision between the robot and the body during target approach. Trajectories that have an angle to the normal vector of the CT table larger than $30^{\circ}$ are therefore dismissed. For all remaining vertices, the insertion angle is stored to be used for rating later.

Next, vertices that produce trajectories that are closer than $3 \mathrm{~mm}$ to bony structures or even collide with bones are excluded by performing a collision detection using the previously generated OBB trees. The same test is performed for already planned trajectories to avoid collisions between needles.

\section{Selection of the best entry point and calculation of end-effector rotation}

All remaining vertices are valid entry points for the defined target point. The vertex with an insertion angle closest to the normal vector of the CT table is selected as the final entry point.

In the last step, the best orientation of the robot endeffector is determined. After needle placement, the needle grasper releases the needle and then moves away from the needle and back to the parking position. To avoid collisions between the robot and the body during this motion, a bounding box modeling a safety zone around the robot is loaded and checked for collisions with the surface model as well as with other placed needles (Fig. 4). If collisions occur, the robot end-effector is rotated by $45^{\circ}$ around the needle axis and tested again, until a valid rotation is found. If all rotations result in collisions, the next best vertex is checked until a valid one is found. When trajectories are altered manually by the user, new collisions are being detected and displayed, but not automatically resolved.

The algorithm is multithreaded, allowing for the planning of additional trajectories while calculations are carried out. It uses all available Central Processing Unit (CPU) cores except one, which is reserved for the graphical user interface.

For the test dataset consisting of 751 slices, the data preparation took $35 \mathrm{~s}$ on a Dell Precision m6800 workstation laptop with a Core i7 processor (Dell Inc., Round Rock, Texas, USA). The data preparation is not multithreaded.

In addition, we assessed the average time required to calculate a trajectory by planning 20 target points in the lung, liver, heart, and kidney and measuring the required time. Calculating one entry point took $9.8 \mathrm{~s}$ on average (4.1-24.1 s, depending on the insertion depth and placement).

\section{Discussion}

In this article, we present an algorithm that allows for the automatic planning of entry points with respect to a given target point for post-mortem robotic needle placement. The automatic planning of entry points removes the most difficult and time-consuming portion of the trajectory planning workflow. This makes it easier to deploy the robot on a routine basis and opens up new research possibilities. An example could be the automated tissue extraction of larger numbers of samples for histological and toxicological studies [15]. The algorithm also minimizes the risk of manually planning a trajectory that leads to collisions and therefore decreases the accuracy or even damages the system. Planning multiple trajectories further increases the collision complexity and can potentially increase the occurrence of such incidents.

These issues can be reduced by using automatic trajectory planning. The value of such an algorithm is supported by a study conducted by Shamir et al. [8], who demonstrated that automatic trajectory planning can reduce the time required for intervention planning and can minimize the trajectory risk for needle insertion into the brain.

As long as needles are placed in the same order that they were planned to be, needle collisions should not occur with the algorithm presented here. In case the needle placement order or needle trajectories are altered manually by the user, collisions should be rechecked, and the end-effector rotation should be recalculated.

On one hand, post-mortem needle placement is easier to handle than clinical needle placement. Better image quality, lower required accuracy, no motion artifacts, and no constraints in terms of critical structures allow for simpler constraints.

On the other hand, time-consuming semi-automatic or even manual segmentation strategies for surfaces, bones, and sensitive structures are not practical in a forensic setting and should be performed automatically. Body surface 
Fig. 4 Needle release motion. After placement of the introducer needle, the gripper releases the needle, moves backwards and slightly upwards, and finally returns the robot to a parking position

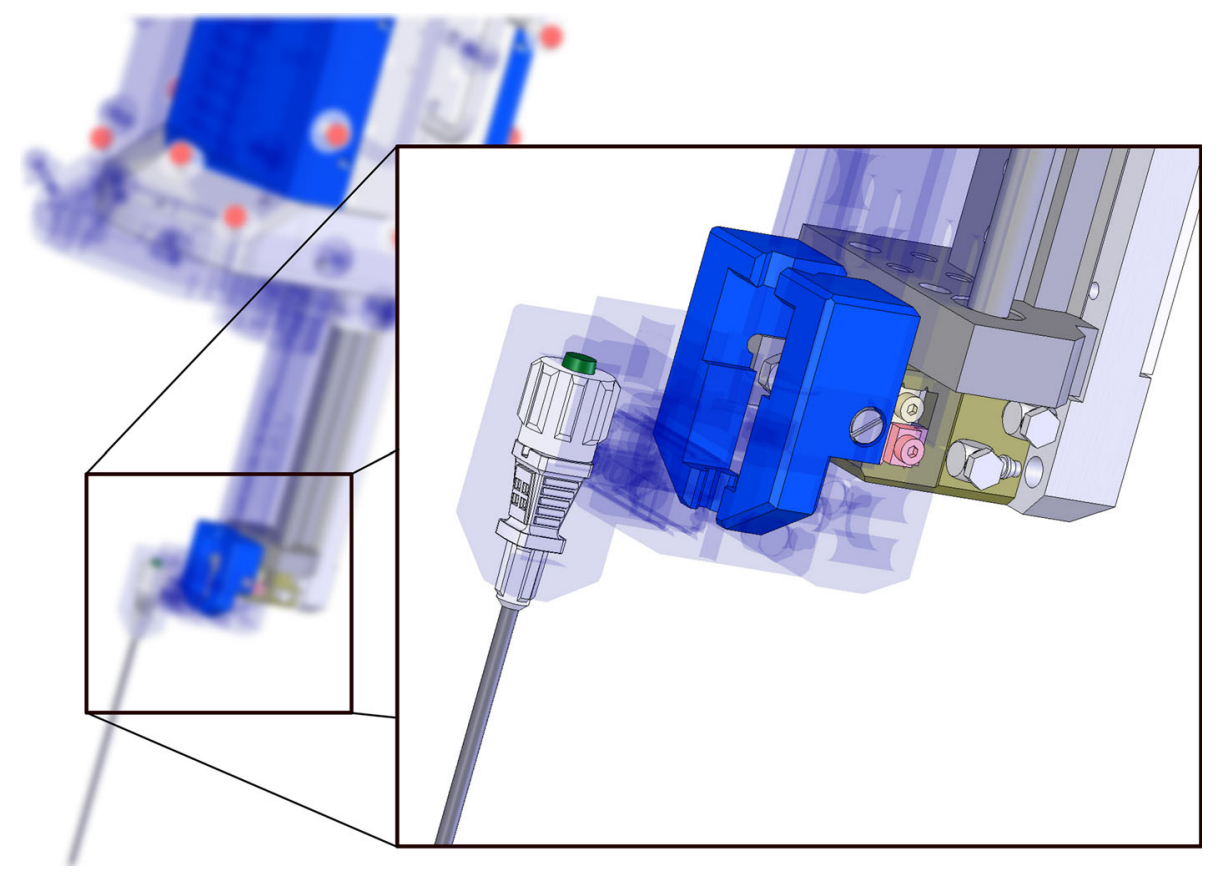

segmentation by thresholding produces satisfying surface models, despite the reduction in image quality due to resampling. As a drawback, threshold segmentation also models internal gas-filled structures such as the lungs and intestines as well as emphysema, gas embolisms, or accumulations of decompositional gas that are very common in a forensic caseload. The presented algorithm addresses this issue by adding another hard constraint that removes these internal vertices. Automatic segmentation of the rib cage and costal cartilage is usually not optimal. In bodies with low bone density (e.g. due to osteoporosis) in particular, the current segmentation algorithm can fail to detect all structures sufficiently, which might cause collisions with bones. In addition, narrow spaces in the bones such as the interlaminar space as access to the spinal canal can be closed due to the downsampling of the dataset, reducing the usability of tasks such as the collection of cerebrospinal fluid. These issues could be minimized by applying more sophisticated segmentation algorithms [16].

Overall, in most cases, the algorithm is sufficiently fast with approximately 5-6 s per entry point, excluding the data preparation that only has to be performed once. This is the case if there is no collision between the end-effector and the body. If the end-effector has to be rotated to avoid collision, calculation times can increase up to $24 \mathrm{~s}$ due to the inefficient collision detection used here. In the future, this process could be sped up by also implementing the use of OBB trees here. In general, calculation times could be sped up by using multithreading for data preparation as well as a Graphics Processing Unit (GPU)-based implementation for collision detection, as demonstrated by Seitel et al. [7]. In general, the actual calculation times play a minor role because they are performed in the background while additional target points can already be defined.

Currently, needles are placed in the same order that they are planned. This means that the manual alteration of an already planned entry point can lead to collisions with other trajectories, which, in the current state, still have to be resolved manually.

Currently, reachability is not a hard constraint, but rather it is tested once after entry point calculation. In rare cases, the robot cannot access a target due to the collinear alignment of two or more robot axes (singularities) during target approach. This can lead to an abortion of the needle placement during the positioning phase of the robot, even if the final position is valid.

To summarize, we developed an algorithm that automatically plans a valid, collision-free needle trajectory for a given target point for post-mortem CT-guided robotic needle placement.

\section{Key points}

1. A trajectory for robotic post-mortem needle placement consists of entry point, target point, and orientation of the biopsy module.

2. Trajectory planning can be time consuming because a variety of boundary conditions have to be considered.

3. An algorithm that automatically calculates the optimal entry point and biopsy module orientation for a given target point is presented. 
4. The algorithm decreases the time required for planning post-mortem robotic CT-guided needle trajectories.

\section{References}

1. Aghayev E, Thali MJ, Sonnenschein M, Jackowski C, Dirnhofer R, Vock P. Post-mortem tissue sampling using computed tomography guidance. Forensic Sci Int. 2007;166:199-203.

2. Hyodoh H, Shimizu J, Mizuo K, Okazaki S, Watanabe S, Inoue H. CT-guided percutaneous needle placement in forensic medicine. Leg Med. 2015;17:79-81.

3. Martinez RM, Ptacek W, Schweitzer W, Kronreif G, Fürst M, Thali MJ, et al. CT-guided, minimally invasive, postmortem needle biopsy using the B-Rob II needle-positioning robot. J Forensic Sci. 2014;59:517-21.

4. Ebert LC, Ptacek W, Naether S, Fürst M, Ross S, Buck U, et al. Virtobot-a multi-functional robotic system for 3D surface scanning and automatic post mortem biopsy. Int $\mathrm{J}$ Med Robot Comput Assist Surg. 2010;6:18-27.

5. Ebert LC, Ptacek W, Breitbeck R, Fürst M, Kronreif G, Martinez RM, et al. Virtobot : the future of automated surface documentation and CT-guided needle placement in forensic medicine. Forensic Sci Med Pathol. 2014;10:179-86.

6. Essert C, Haegelen C, Lalys F, Abadie A, Jannin P. Automatic computation of electrode trajectories for deep brain stimulation: a hybrid symbolic and numerical approach. Int J Comput Assist Radiol Surg. 2011;7:517-32.

7. Seitel A, Engel M, Sommer CM, Radeleff BA, Essert-Villard C, Baegert $\mathrm{C}$, et al. Computer-assisted trajectory planning for percutaneous needle insertions. Med Phys. 2011;38:3246-59.
8. Shamir RR, Joskowicz L, Antiga L, Foroni RI, Shoshan Y. Trajectory planning method for reduced patient risk in imageguided neurosurgery: concept and preliminary results. 2010. p. 76250I-76250I-8. 10.1117/12.843991. Accessed 6 Jan 2016.

9. Pham DL, Xu C, Prince JL. Current methods in medical image segmentation. Annu Rev Biomed Eng. 2000;2:315-37.

10. Shah S, Kapoor A, Ding J, Guion P, Petrisor D, Karanian J, et al. Robotically assisted needle driver: evaluation of safety release, force profiles, and needle spin in a swine abdominal model. Int $\mathrm{J}$ Comput Assist Radiol Surg. 2008;3:173-9.

11. Perry TS. Profile: veebot [Resources_Start-ups]. IEEE Spectr. 2013;50:23.

12. Adams R, Bischof L. Seeded region growing. IEEE Trans Pattern Anal Mach Intell. 1994;16:641-7.

13. Lorensen WE, Cline HE. Marching cubes: A high resolution 3D surface construction algorithm. In: Proceedings of the 14th annual conference on computer graphics and interactive techniques. New York, NY: ACM; 1987. p. 163-169. 10.1145/37401. 37422. Accessed 30 April 2015.

14. Gottschalk S, Lin MC, Manocha D. OBBTree: a hierarchical structure for rapid interference detection. In: Proceedings of the 23rd annual conference on computer graphics and interactive techniques. New York, NY: ACM; 1996. p. 171-180. 10.1145/ 237170.237244. Accessed 30 April 2015.

15. Staeheli SN, Gascho D, Fornaro J, Laberke P, Ebert LC, Martinez RM, et al. Development of CT-guided biopsy sampling for timedependent postmortem redistribution investigations in blood and alternative matrices - proof of concept and application on two cases. Anal Bioanal Chem. 2016;4:1249-58.

16. Banik S, Rangayyan RM, Boag GS. Automatic segmentation of the ribs, the vertebral column, and the spinal canal in pediatric computed tomographic images. J Digit Imaging. 2010;23:301-22. 